\title{
Evaluación de tres pruebas para el diagnóstico de geohelmintos en Colombia
}

\author{
Myriam Consuelo López ${ }^{1}$, Ligia Inés Moncada1 ${ }^{1}$ Yoseth Ariza-Araújo ${ }^{1,2}$, \\ Julián Alfredo Fernández-Niño1, Patricia Reyes', Rubén Santiago Nicholls ${ }^{1,3}$ \\ 1 Departamento de Salud Pública, Facultad de Medicina, Universidad Nacional de Colombia, Bogotá, D.C., \\ Colombia \\ 2 Facultad de Ciencias de la Salud, Universidad Icesi, Cali, Colombia \\ ${ }^{3}$ Organización Panamericana de la Salud, Washington, D.C., Estados Unidos
}

Introducción. Las geohelmintiasis son un problema de salud pública en países en vía de desarrollo. Los estudios de diagnóstico a nivel individual, así como los estudios de población, deben incorporar evaluaciones de su validez y fiabilidad.

Objetivo. Comparar el examen directo, el método de Ritchie-Frick modificado, el de Kato-Katz brasilero y el de Kato-Katz de la OMS, para el diagnóstico de geohelmintos.

Materiales y métodos. Se llevó a cabo un estudio de fiabilidad de pruebas diagnósticas. La misma muestra de materia fecal fue analizada por el mismo observador, empleando cuatro pruebas. Se recolectaron 204 muestras, de las cuales se analizaron 194 que cumplían los criterios de inclusión. Los observadores no conocían la identidad de los participantes, ni el resultado en las otras pruebas. Para el análisis se tomó como método de referencia, la prueba de Kato-Katz de la OMS. Para evaluar la fiabilidad se emplearon el porcentaje de concordancia, el porcentaje de concordancia positiva, la prueba estadística kappa y el coeficiente de correlación intraclase.

Resultados. Se encontró un buen desempeño de la prueba brasilera de Kato-Katz, con alta sensibilidad y especificidad para Trichuris trichiura y Uncinaria sp., 0,97 y 0,96 respectivamente, en ambos valores, y alta especificidad y moderada sensibilidad para Ascaris lumbricoides (0,95 y 0,79), y un desempeño entre moderado y pobre para la prueba de Ritchie-Frick y el examen directo. Las diferencias fueron mayores para Uncinaria sp. y T. trichiura, que para A. lumbricoides.

Conclusión. La prueba brasilera de Kato-Katz podría ser implementada, pero se necesitan más estudios para considerar la relación de su capacidad operativa con su factibilidad, disponibilidad y costo.

Palabras clave: helmintiasis/diagnóstico, salud pública, Colombia

doi: http://dx.doi.org/10.7705/biomedica.v33i1.633

\section{Evaluation (assessment) of three tests for diagnosis of geohelmints in Colombia}

Introduction. Soil-transmitted helminth infections are considered a public health problem in developing countries. The diagnostic tests, both for individual parient diagnosis as for population studies should be evaluated in terms of validity and reliability.

Objective. To compare the direct examination, the modified Ritchie-Frick method, a Kato-Katz designed by a Brazilian group and one designed by the WHO, for the diagnosis of soil-transmitted helminthes.

Materials and methods. A diagnostic test reliability study was performed. The same stool sample was analyzed by the same observer using four diagnostic tests. 204 samples were obtained, 194 of those fulfilled the inclusion criteria and were analyzed. The observers did not know the participants' identity neither the other tests results. For the analysis the Kato-Katz (WHO) was considered as the gold standard. For the reliability assessment percent agreement, positive percent agreement, Kappa statistic, and intraclass correlation were performed.

Results. The Brazilian Kato-Katz showed a good performance with high sensitivity and specificity for T. trichiura and Hookworm with values of 0.97 and 0.96 respectively, and a high specificity with mild sensitivity for $A$. lumbricoides $(0.95$ and 0.79$)$ meanwhile the direct examination and the Ritche-Frick method showed a performance between mild and poor. The differences were higher for hookworm and Trichiuris trichiura than for Ascaris lumbricoides.

\footnotetext{
Contribución de los autores:

Todos los autores participaron en la concepción, diseño del trabajo, análisis e interpretación de resultados, redacción del manuscrito y su aprobación final.
} 
Conclusion. The Brazilian Kato Katz test could be implemented, but further studies are needed to correlate its operative capacity with its feasibility, availability and cost.

Keywords: Helminthiasis/diagnosis, public health, Colombia

doi: http://dx.doi.org/10.7705/biomedica.v33i1.633

Las geohelmintiasis persisten como un problema de salud pública en los países en desarrollo, en donde afectan principalmente a la población infantil. Se estima que en el mundo 2.000 millones de individuos se encuentran infectados por geohelmintos y, de ellos, 300 millones sufren morbilidad grave asociada a las geohelmintiasis, como anemia, desnutrición, déficit cognitivo y trastornos del crecimiento y desarrollo (1-3).

Las frecuencias de geohelmintos en Colombia todavía permanecen altas en algunas regiones del país. Es el caso particular de la población de estudio, en la que desde 1995 se han realizado varias encuestas sobre parasitismo intestinal. La última, de 2005, encontró que la prevalencia de las geohelmintiasis en menores de 18 años que se encuentran escolarizados fue de $24 \%$,y la especie más frecuente fue $A$. lumbricoides, con $21 \%$ (4).

Para planear y evaluar cualquier intervención efectiva de control de las geohelmintiasis, así como de cualquier problema de salud pública, uno de los insumos fundamentales es el conocimiento válido y preciso de su situación epidemiológica. En general, la información disponible sobre frecuencia, distribución y gravedad de una enfermedad se basa principalmente en el rendimiento y las características de las pruebas empleadas para el diagnóstico rutinario o para programas de control. Por esta razón, tanto los estudios de diagnóstico a nivel individual como los estudios de población para el desarrollo de estrategias de control, deberían incorporar evaluaciones de la validez y la fiabilidad de las pruebas diagnósticas empleadas (5).

La técnica de Kato-Katz es el método recomendado por la Organización Mundial de la Salud (OMS) para el diagnóstico, cualitativo y cuantitativo, y el seguimiento de las infecciones intestinales humanas por geohelmintos $(6,7)$. Sin embargo, en la Encuesta Nacional de Morbilidad (8) y en los estudios

\footnotetext{
Correspondencia:

Myriam Consuelo López, Laboratorio de Parasitología, Departamento de Salud Pública, Facultad de Medicina, Universidad Nacional de Colombia, Carrera $30 \mathrm{~N}^{\circ}$ 45-03, Edificio Facultad de Medicina, oficina 471, tercer piso, Ciudad Universitaria, Bogotá, D.C, Colombia

Telefax: (571) 316 5000; extensión 15033

mclopezp@unal.edu.co
}

Recibido: 25/01/12; aceptado:25/08/12 desarrollados en algunas regiones de Colombia, la prueba empleada para el diagnóstico parasitológico fue el método modificado de Ritchie-Frick (9). Por otro lado, en la actualidad hay disponible una prueba de fabricación brasilera que requiere ser evaluada en comparación con la de Kato-Katz de la OMS para considerar su aplicación operativa.

Por lo anterior, en este estudio se comparan las técnicas de examen directo, la modificada de Ritchie-Frick y la de Kato-Katz de fabricación brasilera, con la de Kato-Katz recomendada por la OMS, considerando esta última como la de referencia

\section{Materiales y métodos}

\section{Tipo de estudio}

El presente es un estudio de fiabilidad de pruebas diagnósticas. Se comparó la variabilidad de la determinación de infecciones de parásitos intestinales realizada en la misma muestra, por el mismo observador, usando las siguientes técnicas: el examen directo, la modificada de Ritchie-Frick, la de Kato-Katz (Brasil) y el kit para la de KatoKatz recomendada por la OMS, considerando esta última como el método de referencia.

\section{Área y población de estudio}

El estudio se llevó a cabo en la Inspección de La Virgen, municipio de Quipile (Cundinamarca, Colombia). La inspección de La Virgen está localizada a $4^{\circ} 45^{\prime}$ de latitud norte y $74^{\circ} 32^{\prime}$ de longitud oeste; se encuentra a una altitud de $1.443 \mathrm{msnm}$ y registra una temperatura promedio de $22{ }^{\circ} \mathrm{C}$. La inspección de La Virgen tiene 2.055 habitantes, de los cuales $300(14,6 \%)$ residen en la cabecera municipal y el resto se distribuyen en ocho veredas (Plan de Desarrollo del Municipio de Quipile, 2008-2011).

\section{Tamaño de muestra}

El cálculo del tamaño de muestra se hizo para determinar el mínimo número de participantes requerido para detectar un coeficiente kappa estadísticamente significativo, de acuerdo con la prevalencia obtenida en estudios previos desarrollados en el área de estudio (10). Las condiciones tenidas en cuenta para la selección del tamaño muestral, fueron las siguientes: prevalencia 
de positivos para cualquier geohelminto de $10 \%$, poder estadístico de $90 \%$, significancia estadística de 0,05 , magnitud del coeficiente kappa que se desea detectar de 0,90, valor del coeficiente kappa asumido en la hipótesis nula de 0,60. El tamaño de muestra obtenido fue de 200 participantes.

\section{Diseño de la muestra}

Inicialmente se propuso desarrollar un muestreo probabilístico, sin reemplazo, en una población finita y representativa de cada una de las escuelas que funcionan en el área de estudio (11). Sin embargo, dadas las difíciles condiciones logísticas del trabajo de campo, se solicitó a las directivas de cada institución el listado de estudiantes actualizado a 2010 y se invitó a participar a todos los individuos que voluntariamente desearan recolectar la muestra de materia fecal. El proceso de recolección de muestras e inclusión de individuos, se continuó hasta completar el tamaño de la muestra. Los participantes incluidos en el estudio debieron cumplir con los siguientes criterios de selección.

Criterios de inclusión: niños entre 5 y 15 años de edad que estuvieran matriculados y asistieran regularmente a alguna de las instituciones educativas participantes y de quienes se contara con el consentimiento informado de los padres o los representantes legales y con el asentimiento del propio participante en el caso de los mayores de nueve años.

Criterio de exclusión: el único criterio de exclusión fue presentar alguna contraindicación médica para la administración de cualquiera de los fármacos indicados para el tratamiento de las parasitosis intestinales producidas por helmintos o protozoarios.

\section{Diagnóstico parasitológico}

Se recolectó una muestra de materia fecal de la deposición de la mañana de cada uno de los participantes incluidos en el estudio. Luego de identificadas en el campo, el mismo día de la recolección fueron transportadas al Laboratorio de Parasitología de la Facultad de Medicina de la Universidad Nacional de Colombia, donde se practicaron las siguientes pruebas: examen directo, método de Kato-Katz (con el kit de la OMS) (Vestergaard Frandsen) $(6,12)$, método de KatoKatz (con el kit de fabricación brasilera) (Fundación Oswaldo Cruz-Helm Teste Bio-Manghinhos) y método modificado de concentración de RitchieFrick (9).
Los exámenes directos con solución salina y lugol, se procesaron y analizaron antes de las pruebas de concentración. El tiempo transcurrido entre la recolección y la recepción en el laboratorio, osciló entre seis y nueve horas. Por las complejas condiciones logísticas hubo variación en la duración del periodo comprendido entre la llegada de la muestra y su montaje para la lectura, que osciló entre uno y tres días. Las muestras fueron identificadas con un código único. El montaje de las muestras fue hecho en todas las ocasiones por la misma persona y su lectura la hicieron dos profesionales expertas que no conocían la identidad de los pacientes de quienes se obtuvieron las muestras.

Las muestras fueron procesadas por grupos y se alternó sistemáticamente el montaje y la lectura de tales grupos. En ese sentido, ambas pruebas, la de Kato-Katz de la OMS y la de Kato-Katz de Brasil, se procesaron primero en igual número de veces, siguiendo las recomendaciones de los fabricantes. Puesto que la prueba modificada de Ritchie-Frick emplea formol como conservante, y este permite mantener la morfología de los huevos, quistes y larvas por largos periodos, las muestras fueron analizadas varios días después de su recolección, luego de completarse el análisis de las muestras mediante las pruebas de Kato-Katz.

Una persona externa al grupo de observadores expertos organizó el flujo de muestras que se iban a procesar, de tal manera que los observadores analizaron la misma muestra con las distintas técnicas sin conocer la identidad del sujeto que aportó la muestra o el resultado de los otros métodos empleados. Los resultados fueron reportados para cada código de identificación y solo una de las personas que hicieron el análisis estadístico conocía el nombre y los resultados correspondientes a cada muestra.

\section{Análisis estadísticos}

Para todos los análisis estadísticos se empleó el programa Stata $11^{\mathrm{TM}}$. La prueba considerada como de referencia para las comparaciones fue el kit recomendado por la OMS. Para evaluar la fiabilidad se emplearon el porcentaje de concordancia, el porcentaje de concordancia positiva y el estadístico kappa (13). Se calcularon las estimaciones y sus respectivos intervalos de confianza. La magnitud del coeficiente kappa fue calificada según la escala de Altman (14). Puesto que la variable "huevos por gramo de materia fecal" es de naturaleza continua, para evaluar la reproducibilidad del resultado de 
las dos pruebas de concentración con respecto a la de referencia, se analizó la correlación intraclase en los subgrupos de participantes que tenían el diagnóstico de infección por cualquiera de las dos.

\section{Consideraciones éticas}

Esta investigación fue considerada como de riesgo mínimo para los participantes (15). Las consideraciones éticas que guiaron el desarrollo de esta investigación fueron coherentes con la Declaración de Helsinki, la Resolución No. 008430 de 1993 del Ministerio de Salud de Colombia y el código del menor (16). Se obtuvo consentimiento informado escrito de los padres o los representantes legales de los menores y asentimiento para los niños mayores de nueve años. Los participantes en el estudio no recibieron ningún incentivo económico. En los casos requeridos se suministró el tratamiento indicado de acuerdo con los esquemas vigentes y a todos los participantes se les suministró información sobre la infección por geohelmintos.

\section{Resultados}

\section{Descripción de los participantes}

Se logró obtener 204 muestras de materia fecal de igual número de participantes; 194 cumplieron los criterios de inclusión, 92 (47,4 \%) fueron de sexo femenino y 102 (52,6\%) de sexo masculino. La edad mínima en ambos sexos fue 5 años, y la máxima para mujeres fue 13 y para los hombres 14 años.

\section{Frecuencias de detección de cada especie de} geohelminto

Según la prueba de Kato-Katz recomendada por la OMS, considerada como método de referencia, las proporciones de infecciones por A. lumbricoides, $T$. trichiura y Uncinaria sp. fueron, respectivamente, de $22,7 \%$ (44/194), 37,1\% (72/194) y 13,4\% (26/194). La proporción de individuos con, al menos, uno de los tres geohelmintos fue de $50,5 \%$ en la muestra total e igual proporción se encontró al estratificar por sexo. Sólo 7 individuos (3,6 \%), 5 mujeres y 2 hombres, tuvieron simultáneamente infección por los tres geohelmintos. No es posible estimar la prevalencia de población por la forma como fueron seleccionados los participantes. En el cuadro 1 se presenta la comparación de los resultados de las tres pruebas diagnósticas de interés respecto a los resultados reportados con el kit de Kato-Katz de la OMS.

Puesto que el principio para determinar que una prueba es positiva es la observación de, al menos, un huevo del geohelminto, es poco probable que un resultado positivo reportado de cualquiera de las pruebas se trate de un error de clasificación, al tratarse de lectores expertos. Por esta razón, en el cuadro 1 se ha subrayado la frecuencia de un tipo particular de desacuerdo: las muestras reportadas como negativas con la prueba de referencia: el Kato-Kat de la OMS, pero que fueron reportadas como positivas en las pruebas en evaluación. La proporción de pruebas con este resultado varía de un parásito a otro y muestra un incremento a medida que aumenta la sensibilidad de la prueba (cuadro 2). De esta manera, para A. lumbricoides las muestras reportadas como negativas con el Kato-Katz (OMS) que fueron detectados por el examen directo, corresponden al 1,3\%, en el caso del método modificado de Ritchie-Frick, equivalen al 2,7\% y en el caso de la prueba de Kato-Katz de Brasil, al 4,7\%. Al tener en cuenta esta información para la determinación de la frecuencia de las geohelmintiasis en la muestra, se obtienen las siguientes frecuencias: 54 en $A$. lumbricoides (27,7 $\%), 75$ en $T$. trichiura (38,5 \%) y 34 en Uncinaria sp. $(17,4 \%)$.

La valoración de la magnitud del coeficiente kappa según la escala propuesta por Altman (14), permite calificar la concordancia entre las pruebas para

Cuadro 1. Comparación de los resultados de las pruebas examen directo, método modificado de Ritchie Frick y la prueba de Kato Katz del Brasil, frente al método de referencia Kato Katz de la OMS

\begin{tabular}{|c|c|c|c|c|c|c|c|}
\hline \multirow[b]{2}{*}{ Parásito } & \multirow[b]{2}{*}{$\begin{array}{c}\text { Kato-Katz } \\
\text { (OMS) }\end{array}$} & \multicolumn{2}{|c|}{$\begin{array}{l}\text { Examen } \\
\text { directo }\end{array}$} & \multicolumn{2}{|c|}{$\begin{array}{c}\text { Ritchie Frick } \\
\text { modificado }\end{array}$} & \multicolumn{2}{|c|}{$\begin{array}{c}\text { Kato-Katz } \\
\text { (Brasil) }\end{array}$} \\
\hline & & $(+)$ & $(-)$ & $(+)$ & $(-)$ & $(+)$ & $(-)$ \\
\hline \multirow[t]{2}{*}{ Ascaris lumbricoides } & $(+)$ & 17 & 27 & 25 & 19 & 35 & 9 \\
\hline & $(-)$ & 2 & 148 & 4 & 146 & 7 & 143 \\
\hline \multirow[t]{2}{*}{ Trichuris trichiura } & $(+)$ & 16 & 56 & 17 & 55 & 56 & 16 \\
\hline & $(-)$ & 2 & 120 & 2 & 120 & 3 & 119 \\
\hline \multirow[t]{2}{*}{ Uncinaria sp. } & $(+)$ & 3 & 23 & 3 & 23 & 19 & 7 \\
\hline & $(-)$ & 0 & 168 & 1 & 167 & 7 & 161 \\
\hline
\end{tabular}


detectar cada geohelminto (cuadro 2). Según la escala, la concordancia del examen directo con la de Kato-Katz (OMS) es regular para $A$. lumbricoides y mala para $T$. trichiura y Uncinaria sp. La concordancia del método modificado de Ritchie-Frick respecto al de referencia es moderada para A. lumbricoides, regular para $T$. trichiura y mala para Uncinaria sp. La concordancia entre la prueba de Kato-Katz de Brasil y la de Kato-Katz de la OMS se puede considerar buena para los tres geohelmintos analizados.

Las pruebas de Kato-Katz y el método de concentración de Ritchie-Frick permiten la estimación de la carga parasitaria expresada en huevos por gramo de materia fecal. En el cuadro 3 se presenta la distribución de esta variable. Las diferencias entre el número de huevos detectados por la pruebas para cada tipo de parásito, no fueron estadísticamente significativas (prueba del signo de Wilcoxon: $p>0,10$ ).

En el cuadro 4 se presenta la distribución de las concordancias de ambas pruebas según los grados de intensidad de la infección propuestos por la OMS.
En este cuadro, las celdas correspondientes a la concordancia completa por grados de intensidad muestran la frecuencia absoluta. Se observa que el número de concordancias es mayor para el kit de Kato-Katz de fabricación brasilera que para el método modificado de Ritchie-Frick.

Además, se hizo un análisis de correlación intraclase en los subgrupos de participantes que, en efecto, tenían el diagnóstico de infección por cualquiera de las dos pruebas. En el cuadro 5 se presentan los coeficientes intraclases de las cargas parasitarias entre el método modificado de Rithchie-Frick y la prueba de Kato-Katz del Brasil comparada con la de Kato Katz de la OMS, para cada especie de geohelminto y según el grado de infección definido por la prueba de la OMS, considerada como método de referencia.

Las comparaciones de la concordancia de las tres pruebas diagnósticas hechas con la Kato-Katz de la OMS para la infección por cada uno de los parásitos analizados, se presentan finalmente consolidadas en las figuras 1 a 3 . En todos los

Cuadro 2. Comparación de la sensibilidad, especificidad y valores kappa de las pruebas

\begin{tabular}{|c|c|c|c|c|c|c|c|c|c|}
\hline & $\begin{array}{l}\text { Sensibilidad } \\
\text { (IC } 95 \%)\end{array}$ & & & $\begin{array}{c}\text { Especificida } \\
\text { (IC } 95 \% \text { ) }\end{array}$ & & & $\begin{array}{c}\text { Kappa }^{a} \\
\text { (IC } 95 \% \text { \%) }\end{array}$ & & \\
\hline & $\begin{array}{l}\text { Examen } \\
\text { directo }\end{array}$ & $\begin{array}{c}\text { Ritchie- } \\
\text { Frick }\end{array}$ & $\begin{array}{c}\text { Kato-Katz } \\
\text { (Brasil) }\end{array}$ & $\begin{array}{l}\text { Examen } \\
\text { directo }\end{array}$ & $\begin{array}{c}\text { Ritchie- } \\
\text { Frick }\end{array}$ & $\begin{array}{c}\text { Kato-Katz } \\
\text { (Brasil) }\end{array}$ & $\begin{array}{l}\text { Examen } \\
\text { directo }\end{array}$ & $\begin{array}{c}\text { Ritchie- } \\
\text { Frick }\end{array}$ & $\begin{array}{c}\text { Kato-Katz } \\
\text { (Brasil) }^{\mathrm{a}}\end{array}$ \\
\hline Ascaris lumbricoides & $\begin{array}{c}0,39 \\
(0,24-0,54)\end{array}$ & $\begin{array}{c}0,56 \\
(0,41-0,72)\end{array}$ & $\begin{array}{c}0,79 \\
(0,67-0,92)\end{array}$ & $\begin{array}{c}0,98 \\
(0,96-1)\end{array}$ & $\begin{array}{c}0,97 \\
(0,94-0,99)\end{array}$ & $\begin{array}{c}0,95 \\
(0,92-0,99)\end{array}$ & $\begin{array}{c}0,46 \\
(0,31-0,62)\end{array}$ & $\begin{array}{c}0,61 \\
(0,48-0,76)\end{array}$ & $\begin{array}{c}0,76 \\
(0,65-0,87)\end{array}$ \\
\hline Trichuris trichiura & $\begin{array}{c}0,22 \\
(0,12-0,32)\end{array}$ & $\begin{array}{c}0,24 \\
(0,13-0,34)\end{array}$ & $\begin{array}{c}0,97 \\
(0,94-1)\end{array}$ & $\begin{array}{c}0,98 \\
0,98(0,96-1)\end{array}$ & $\begin{array}{c}0,98 \\
(0,96-1)\end{array}$ & $\begin{array}{c}0,97 \\
(0,94-1)\end{array}$ & $\begin{array}{c}0,24 \\
(0,13-0,35)\end{array}$ & $\begin{array}{c}0,26 \\
(0,15-0,38)\end{array}$ & $\begin{array}{c}0,78 \\
(0,69-0,87)\end{array}$ \\
\hline Uncinaria spp. & $\begin{array}{c}0,11 \\
(-0,1-0,24)\end{array}$ & $\begin{array}{c}0,11 \\
(0,02-0,25)\end{array}$ & $\begin{array}{c}0,96 \\
(0,92-0,98)\end{array}$ & $\begin{array}{c}1 \\
(\mathrm{NA})\end{array}$ & $\begin{array}{c}0,99 \\
(0,98-1)\end{array}$ & $\begin{array}{c}0,96 \\
(0,92-0,98)\end{array}$ & $\begin{array}{c}0,18 \\
(0,003-0,36)\end{array}$ & $\begin{array}{c}0,17 \\
(-0,009-0,35)\end{array}$ & $\begin{array}{c}0,69 \\
(0,54-0,84)\end{array}$ \\
\hline
\end{tabular}

a Todos los cálculos de kappa obtenidos fueron estadísticamente significativos $(p<0,001)$

Cuadro 3. Intensidad de infección por geohelmintos estimada por tres pruebas diagnósticas en escolares de 5 a 15 años residentes en zona rural de Cundinamarca, Colombia, 2010

\begin{tabular}{|c|c|c|c|c|c|}
\hline \multirow[t]{2}{*}{ Parásito } & \multirow[t]{2}{*}{ Prueba } & \multirow[t]{2}{*}{$\begin{array}{c}\text { Muestras positivas } \\
\%\end{array}$} & \multicolumn{3}{|c|}{$\begin{array}{c}\text { Huevos por gramo } \\
\text { de material fecal }\end{array}$} \\
\hline & & & Mín. & Med. & Máx. \\
\hline \multirow[t]{3}{*}{ Ascaris lumbricoides } & Kato-Katz (OMS) & 22,7 & 24 & 1272 & 622.368 \\
\hline & RFM & 15,0 & 160 & 1920 & 278.880 \\
\hline & Kato-Katz (Brasil) & 21,7 & 24 & 996 & 660.786 \\
\hline \multirow[t]{3}{*}{ Trichuris trichiura } & Kato-Katz (OMS) & 37,1 & 24 & 144 & 53.952 \\
\hline & RFM & 9,8 & 160 & 480 & 24.000 \\
\hline & Kato-Katz (Brasil) & 30,4 & 24 & 312 & 61.320 \\
\hline \multirow[t]{3}{*}{ Uncinaria sp. } & Kato-Katz (OMS) & 13,4 & 24 & 120 & 2448 \\
\hline & RFM & 2,0 & 160 & 240 & 320 \\
\hline & Kato-Katz (Brasil) & 13,4 & 24 & 108 & 1248 \\
\hline
\end{tabular}

RFM: modificada de Ritchie-Frick;

Ninguna diferencia fue estadísticamente significativa, $p>0,10$. 
Cuadro 4. Concordancias de dos pruebas diagnósticas por la intensidad de la infección, según huevos por gramo de heces

\begin{tabular}{|c|c|c|c|c|c|c|c|c|c|}
\hline \multirow[b]{2}{*}{ Geohelminto } & \multirow{2}{*}{$\begin{array}{l}\text { Kato-Katz (OMS) } \\
\text { Intensidad hpg }\end{array}$} & \multicolumn{4}{|c|}{ Método modificado de Ritchie-Frick } & \multicolumn{4}{|c|}{ Kato-Katz (Brasil) } \\
\hline & & Negativo & Leve & Moderada & Grave & Negativo & Leve & Moderada & Grave \\
\hline \multirow[t]{4}{*}{ Ascaris } & Negativo & $146(97)$ & $\begin{array}{c}3 \\
10\end{array}$ & 1 & 0 & $143(95)$ & $\begin{array}{c}7 \\
15\end{array}$ & 0 & 0 \\
\hline & Leve $\quad<5.000$ & 15 & $(40)$ & 0 & 0 & 9 & $(60)$ & 1 & 0 \\
\hline & Moderada $5.000-49999$ & 4 & 4 & $5(38)$ & 0 & 0 & 0 & $12(92)$ & 1 \\
\hline & Grave $>50.000$ & 0 & 1 & 3 & $2(33)$ & 0 & 0 & 1 & $5(83)$ \\
\hline \multirow[t]{4}{*}{ Tricocéfalos } & Negativo & $119(98)$ & 2 & 0 & 0 & $119(98)$ & $\begin{array}{c}3 \\
40\end{array}$ & 0 & 0 \\
\hline & Leve $\quad<1.000$ & 49 & $6(11)$ & 0 & 0 & 16 & $(70)$ & 1 & 0 \\
\hline & Moderada 1.000-9.999 & 4 & 5 & $2(18)$ & 0 & 0 & 1 & $10(91)$ & 0 \\
\hline & Grave $\quad>10.000$ & 0 & 1 & 2 & $1(25)$ & 0 & 0 & 2 & $2(50)$ \\
\hline \multirow[t]{4}{*}{ Uncinaria } & Negativo & $167(99)$ & 1 & 0 & 0 & $161(96)$ & 7 & 0 & 0 \\
\hline & Leve $\quad<2.000$ & 22 & $3(12)$ & 0 & 0 & 7 & $18(72)$ & 0 & 0 \\
\hline & Moderada $2.000-3.999$ & 1 & 0 & $0(0)$ & 0 & 0 & 1 & $0(0)$ & 0 \\
\hline & Grave $>4.000$ & 0 & 0 & 0 & $0(100)$ & 0 & 0 & 0 & $0(100)$ \\
\hline
\end{tabular}

Nota: los números en paréntesis corresponden a la frecuencia relativa en las celdas donde se espera concordancia

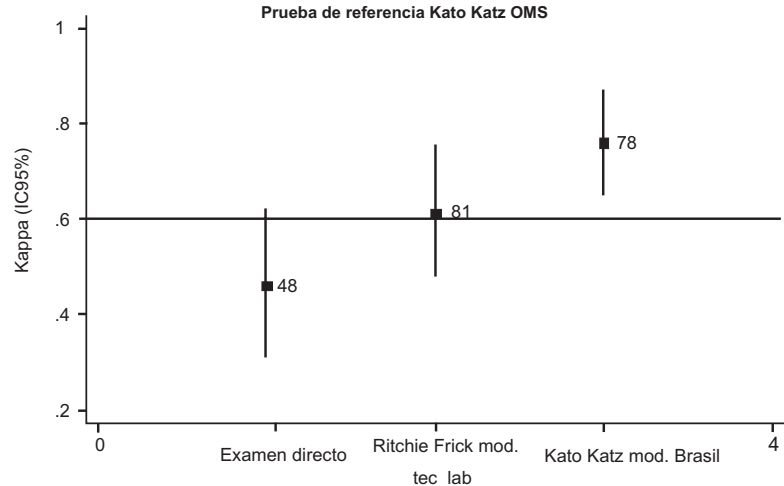

Figura 1. Comparación de la concordancia de las pruebas diagnósticas evaluadas para Ascaris lumbricoides

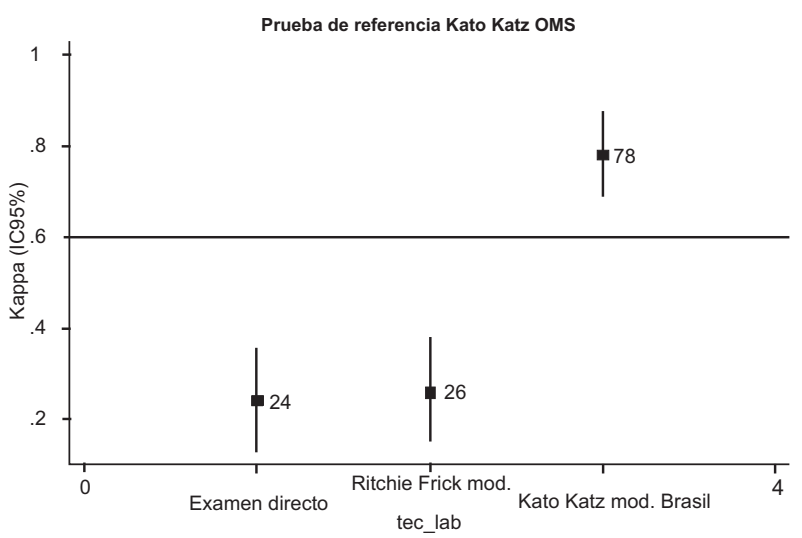

Figura 2. Comparación de la concordancia de las tres pruebas diagnósticas evaluadas para Trichuris trichiura

casos, la prueba de Kato-Katz de Brasil presentó el mejor desempeño.

\section{Discusión}

El proceso de selección de los participantes no fue aleatorio y se incluyeron los que voluntariamente decidieron participar. Esta modificación del protocolo

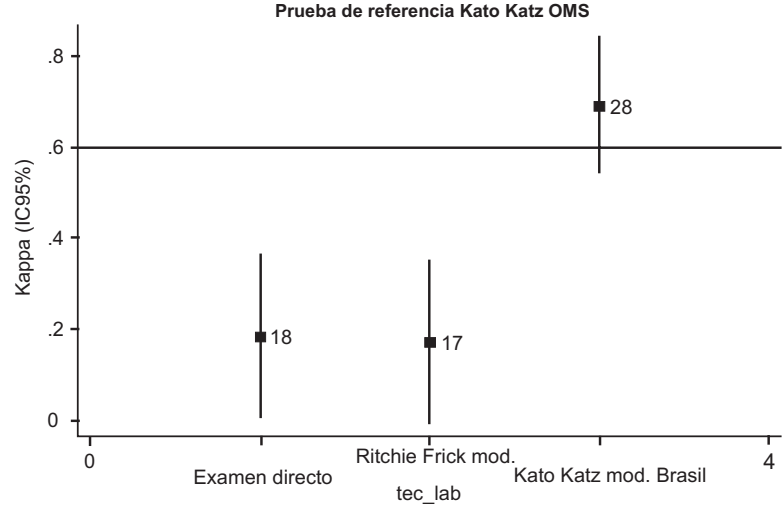

Figura 3. Comparación de la concordancia de las tres pruebas diagnósticas para uncinariasis

originalmente propuesto, no permitió conocer la probabilidad de ingreso a la muestra y, por lo tanto, no fue posible calcular el factor de expansión correspondiente para el cálculo del estimador de interés, la prevalencia. Sin embargo, este hecho no introduce ninguna amenaza a la validez de los resultados obtenidos que están directamente relacionados con la evaluación de la confiabilidad de las pruebas diagnósticas propuesta por este trabajo. Además, se emplearon medidas de control de calidad, enmascaramiento de las muestras y seguimiento estricto de los protocolos establecidos, para minimizar el sesgo de información.

Los resultados indican que la sensibilidad de la prueba de Kato-Katz del Brasil no superó el 80 \% para ninguna especie de geohelminto. Sin embargo, el valor calculado presentó diferencias para cada especie de geohelminto, siendo mayor para $A$. lumbricoides (79\%), en contraste con T. trichiura (77\%) y Uncinaria sp. (73 \%). Por el contrario, la especificidad de la prueba de fabricación brasilera 
Cuadro 5. Correlación entre clases entre la intensidad de la infección por geohelmintos estimado por el método modificado de Ritchie Frick y la prueba brasilera de Kato-Katz comparada con la de Kato-Katz de la OMS en escolares de 5 a 15 años residentes en la zona rural de Cundinamarca, Colombia, 2010

\begin{tabular}{|c|c|c|c|c|c|c|}
\hline \multirow[b]{2}{*}{ Geohelminto } & \multirow[b]{2}{*}{$\begin{array}{l}\text { Intensidad } \\
\text { de la infección }\end{array}$} & \multirow[b]{2}{*}{$\mathbf{n}$} & \multicolumn{2}{|c|}{ Método modificado de Ritchie-Frick } & \multicolumn{2}{|l|}{ Kato-Katz (Brasil) } \\
\hline & & & $\begin{array}{l}\text { Coeficiente de } \\
\text { correlación intraclase }\end{array}$ & $p$ & $\begin{array}{c}\text { Coeficiente de } \\
\text { correlación intraclase }\end{array}$ & p \\
\hline \multirow[t]{4}{*}{ Áscaris } & Total & 54 & 0,15 & $<0,001$ & 0,94 & $<0,001$ \\
\hline & Leve & 25 & 0,72 & $<0,001$ & 0,87 & $<0,001$ \\
\hline & Moderada & 13 & 0,25 & 0,41 & 0,87 & $<0,001$ \\
\hline & Grave & 6 & $-0,08$ & 0,87 & 0,77 & 0,07 \\
\hline \multirow[t]{4}{*}{ Tricocéfalos } & Total & 72 & 0,54 & $<0,001$ & 0,85 & $<0,001$ \\
\hline & Leve & 57 & 0,16 & 0,228 & 0,71 & $<0,001$ \\
\hline & Moderada & 11 & $-0,16$ & 0,631 & 0,08 & 0,8 \\
\hline & Grave & 4 & 0,8 & 0,2 & 0,8 & 0,2 \\
\hline \multirow[t]{4}{*}{ Uncinarias } & Total & 26 & $-0,009$ & 0,964 & 0,56 & $<0,005$ \\
\hline & Leve & 25 & 0,01 & 0,943 & 0,5 & 0,01 \\
\hline & Moderado & 1 & NA & NA & NA & NA \\
\hline & Grave & 0 & NA & NA & NA & NA \\
\hline
\end{tabular}

NA: no aplica

fue alta con pequeñas diferencias entre especies de geohelmintos, con valores de $95 \%$ para $A$. lumbricoides, de $97 \%$ para T. trichiura y de $96 \%$ para Uncinaria sp. En general, la especificidad fue también alta para las demás pruebas evaluadas ya que el problema de este tipo de examen diagnóstico, al menos cuando se cuenta con lectores entrenados, es eminentemente de sensibilidad.

Según la clasificación de Altman (14), los valores de kappa obtenidos entre la prueba de Kato-Katz del Brasil y la de Kato-Katz de la OMS -para todos los parásitos- son considerados como "buenos" por hallarse entre 0,6 y 0,8 , es decir, evidencian que la Kato-Katz del Brasil tiene, en general, un buen desempeño. El coeficiente kappa ofreció mayor ventaja sobre otras aproximaciones porque su resultado incorpora una corrección que se ajusta por la concordancia debida al azar.

Con respecto a la correlación entre el número de huevos por gramo detectado por la Kato-Katz de la OMS y la brasilera, fue posible observar que el grado de correlación es positivo y directo. Sin embargo, varía de un parásito a otro: es mayor en A. lumbricoides $(0,97)$ y alcanza un poco más de 0,50 en Uncinaria sp. La correlación observada es heterogénea dentro de cada una de las infecciones parasitarias según la intensidad de la infección, y es mayor en las infecciones de intensidad leve. Sin embargo, este hallazgo debe analizarse con cautela, pues las categorías de infecciones moderadas e intensas tienen menor número de participantes, lo que limita la contundencia de las conclusiones.
Por su parte, los valores de kappa entre la prueba de Kato-Katz de la OMS y el método de RitchieFrick según la escala de Altman, se consideran "moderados, regulares y bajos" para A. lumbricoides, T. trichiura y Uncinaria sp., respectivamente. Los valores de kappa entre la prueba de Kato-Katz de la OMS y el examen directo según la escala de Altman, se consideran "moderados, regulares y malos" para A. lumbricoides, T. trichiura y Uncinaria sp., respectivamente.

Laveche, et al. (17), compararon cualitativa y cuantitativamente los métodos de McMaster y la Kato-Katz, y encontraron resultados similares a los del presente artículo, en que el método de KatoKatz de la OMS fue más sensible para la detección de $A$. lumbricoides y, para $T$. trichiura y Uncinaria sp., las diferencias no fueron significativas.

Los resultados ratifican que el examen directo y el método de Ritchie-Frick tienen, en general, un desempeño entre regular y bajo como pruebas diagnósticas para geohelmintos con mucho menos concordancia para Uncinaria sp. Por el contrario, la prueba de Kato-Katz de Brasil, como ya se señaló, tiene una buena concordancia con la de Kato-Katz de la OMS. Estos resultados concuerdan con los de Muñoz, et al. (18), al comparar la Kato-Katz de la OMS con el método modificado de concentración de Ritchie-Frick.

En otro estudio llevado a cabo en el municipio de Apartadó (Antioquia, Colombia), Arboleda, et al. (19), informaron que las frecuencias de geohelmintos diagnosticadas mediante el examen directo fueron menores que las halladas por la prueba de Kato- 
Katz. En los estudios realizados en zonas donde se están desarrollando programas de control de las geohelmintiasis basados en quimioterapia masiva, se recomienda el análisis previo de muestras seriadas y la utilización de varias pruebas diagnósticas (4). En un estudio llevado a cabo en Zanzíbar, se analizaron muestras de heces de 279 escolares con la prueba de Kato-Katz y la técnica de FLOTAC. Se consideró como método de referencia la suma de los resultados de ambas técnicas. Las sensibilidades de la técnica FLOTAC para el diagnóstico de T. trichiura, Uncinaria sp. y A. lumbricoides, fueron de 88,7, 83,0 y $82,8 \%$, respectivamente $(19,20)$. Se reportaron valores inferiores de sensibilidad para el método de Kato-Katz: $71,8,46,0$ y 70,3 \%, respectivamente (12). Se encontró que el FLOTAC tenía una mayor sensibilidad que el de Kato-Katz por duplicado $(21,22)$. Sin embargo, en la práctica clínica individual y en la implementación de actividades colectivas, habitualmente se analiza una sola muestra. Este razonamiento fue empleado en nuestro estudio para definir el número de muestras por participante.

Los resultados muestran que las características de las pruebas varían según la especie de geohelminto. Un resultado constante es que la diferencia entre las pruebas evaluadas y el estándar es mayor en el caso de Uncinaria sp. A este respecto, Dacombe, et al. (23), encontraron que después de transcurridas tres horas entre la recolección y el análisis de la muestra, hay un descenso de casi $50 \%$ en la sensibilidad, tanto para la Kato-Katz como para la técnica de concentración de formol éter. Por tanto, es recomendable que la muestra se procese dentro de las tres primeras horas después de emitida la muestra, tanto por las técnicas de Kato-Katz, como por otros métodos.

Machicado, et al. (24), compararon la técnica de sedimentación espontánea en tubo de centrífuga con la Kato-Katz de la OMS y encontraron que ambas tenían la misma tasa de detección de huevos de geohelmintos, pero la primera tuvo un conteo de huevos más alto y, al mismo tiempo, se pueden observar los protozoarios intestinales, a diferencia de la Kato-Katz de la OMS.

Los resultados de este trabajo permiten concluir que la Kato-Katz de Brasil tiene un buen desempeño; sin embargo, para su recomendación también deben considerarse la factibilidad técnica y los costos de su implementación. No obstante, la información presentada puede ser útil para evaluar la fiabilidad de los resultados obtenidos en estudios previos y contribuir a la formulación de las recomendaciones de uso de las pruebas evaluadas en condiciones de campo. Finalmente, los resultados de este estudio pueden contribuir a consolidar la información disponible para continuar en la búsqueda de mejorar y simplificar el diagnóstico de los parásitos intestinales: protozoarios y geohelmintos (1).

\section{Agradecimientos}

Agradecemos a Steven Ault, a la bacterióloga Emelina Barraza y a la comunidad de La Virgen.

\section{Conflictos de intereses}

Los autores declaran que no incurrieron en conflictos de intereses durante este estudio.

\section{Financiación}

Este estudio fue cofinanciado por la Organización Panamericana de la Salud y por la Universidad Nacional de Colombia.

\section{Referencias}

1. De Carvalho GL, Moreira LE, Pena JL, Marinho CC, Bahia MT, Machado-Coelho GL. A comparative study of the TF-Test ${ }^{\circledR}$, Kato-Katz, Hoffman-Pons-Janer, Willis and Baermann-Moraes coprologic methods for the detection of human parasitosis. Mem Inst Oswaldo Cruz. 2012;107:80-4. http://dx.doi.org/10.1590/S0074-02762012000100011

2. World Health Organization. Schistosomiasis and soil transmitted helminth infections. WHO, Geneva. Wkly Epidemiol Rec. 2006;81:145-63.

3. De Silva NR, Brooker S, Hotez PJ, Montresor A, Engels D, Savioli L. Soil-transmitted helminth infections: Updating the global picture. Trends Parasitol. 2003;19:547-51. http:// dx.doi.org/10.1016/j.pt.2003.10.002

4. Fernández JA, Reyes P, Moncada LI, López MC, Chaves MP, Knudson A, et al. Tendencia y prevalencia de las geohelmintiasis en La Virgen, Colombia 1995-2005. Rev Salud Pública (Bogotá). 2007;9:289-96. http://dx.doi. org/10.1590/S0124-00642007000200012

5. Szklo M, Nieto J. Epidemiología intermedia: conceptos y aplicaciones. Madrid: Ediciones Díaz de Santos; 2003. p. 395-999.

6. Katz N, Chaves A, Pellegrino J. A simple device for quantitative stool thick-smear technique in Schistosomiasis mansoni. Rev Inst Med Trop Sao Paulo. 1972;14:397-400.

7. World Health Organization. Cellophane faecal thick smear examination technique (Kato) for diagnosis of intestinal schistosomiasis and gastrointestinal helminth infections. PDP/ 83.3, Geneva: WHO; 1983.

8. Cáceres $E$, Castaño L, Estupiñán $D$, López $M C$, Páez $S$, Pinilla CA, et al. En: Corredor A, Arciniegas E, Hernández CA, editores. Parasitismo intestinal. Ministerio de Salud, Instituto Nacional de Salud. Bogotá: Instituto Nacional de Salud; 2000. p. 67-8.

9. Beck JW, García A, Jartog EM, Shaner AL. Empleo de la técnica de recuento de huevos de Ritchie-Frick en el estudio de la efectividad del antihelmíntico nopar (yoduro de stilbazium). Rev Fac Med Unal. 1965;14:33-6. 
10. Kristian L. Necessary sample size for method comparison studies based on regression analysis. Clin Chem. 1999;45:882-94.

11. Silva LC. Muestreo para la investigación en ciencias de la salud. Madrid: Ediciones Díaz de Santos; 1993.

12. Goodman D, Haji HJ, Bickle QD, Stoltzfus RJ, Tielsch JM, Ramsan M, et al. A comparison of methods for detecting the eggs of Ascaris, Trichuris, and hookworm in infant stool, and the epidemiology of infection in Zanzibari infants. Am J Trop Med Hyg. 2007;76:725-31.

13. Sim J, Weight S. The kappa statistic in reliability studies: Use, interpretation, and sample size requirements. Phys Ther. 2005;85:257-68.

14. Altman D, Machin D, Bryant T, Gardner S. Click to close statistics with confidence: Confidence intervals and statistical guidelines, Bristol: British Medical Journal; 2000. p. 256.

15. Ministerio de Salud. Dirección de desarrollo científico y tecnológico. Normas científicas, técnicas y administrativas para la investigación en salud. Resolución No. 008430, Santafé de Bogotá: Ministerio de Salud; 1993.

16. Código del menor. Derechos del niño. Decreto 2737, artículo 13, noviembre 1989.

17. Laveche B, Behnke JM, Ajjampur SS, Albonico M, Ame SM, Charlier J, et al. A comparison of the sensitivity and fecal egg counts of the McMaster Egg counting and Kato-Katz thick smear methods for soil-transmitted helminthes. PLOS Negl Trop Dis. 2011;5:e1201. http://dx.doi.org/10.1371/ journal.pntd.0001201

18. Muñoz G, Camacho M, Silva D, Hincapié M, Gallo E. Dinámica poblacional de Ascaris lumbricoides en una comunidad rural de Floridablanca, Santander, bajo las políticas de control consignadas en la ley 100. Salud UIS. 2004;36:65-72.

19. Arboleda M, Lopera T, Restrepo M, Botero D, Lotero MC, Ríos P. Efectos de la desparasitación comunitaria en la población infantil del área urbana de Apartadó, Colombia. Revista CES Medicina. 2004;18:52-9.

20. Knopp S, Mgeni A, Khamis S, Steinmann P, Stothard R, Rollinson D, et al. Diagnosis of soil-transmitted helminthes in the era of preventive chemotherapy: Effect of multiple stool sampling and use of different diagnostic techniques. PLoS Negl Trop Dis. 2008;2:e331. http://dx.doi.org/10.1371/ journal.pntd.0000331

21. Knopp S, Rinaldi L, Khamis IS, Stothard JR, Rollinson D, Maurelli MP, et al. A single FLOTAC is more sensitive than triplicate Kato-Katz for the diagnosis of low-intensity soil-transmitted helminth infections. Trans $\mathrm{R}$ Soc Trop Med Hyg. 2009;103:347-54. http://dx.doi.org/10.1016/j. trstmh.2008.11.013

22. Knopp S, Speich B, Hattendorf J, Rinaldi L, Mohammed KA, Khamis SI, et al. Diagnostic accuracy of Kato-Katz and FLOTAC for assessing anthelmintic drug efficacy. PLoS Negl Trop Dis. 2011;5:e1036. http://dx.doi.org/10.1371/ journal.pntd.0001036

23. Dacombe RJ, Crampin AC, Flyd S, Randall A, Ndhlovu $\mathbf{R}$, Bickle $\mathbf{Q}$, et al. Time delays between patient and laboratory selectively affect accuracy of helminth diagnosis. Trans R Soc Trop Med Hyg. 2007;101:140-5. http://dx.doi. org/10.1016/j.trstmh.2006.04.008

24. Machicado JD, Marcos LA, Tello R, Canales M. Diagnosis of soil-transmitted helminthiasis in an Amazonic community of Perú using multiple diagnostic techniques. Trans $\mathrm{R}$ Soc Trop Med Hyg. 2012;106:333-9. http://dx.doi.org/10.1016/j. trstmh.2012.03.004 